\title{
Sensitivity analysis of the metric based test selection
}

\author{
Jadranka A. Curgus \\ Boeing ISS Research $\mathbb{E}$ Technology \\ Bellevue, WA, USA \\ Son T. Vuong, Jinsong Zhu \\ Department of Computer Science \\ University of British Columbia \\ Vancouver, B.C., Canada V6T 124 \\ Email: $\{v u o n g, j z h u\} @ c s . u b c . c a$
}

\begin{abstract}
In our earlier work [7, 3], we introduced a novel concept of test distance and an effective multi-pass metric-based method of test selection for communication protocols. The main weight of the method rests on the concept of test distance, which forms the basis of a metric definition to guide the convergent test selection process. In this paper, we discuss a sensitivity analysis of this metric based test selection method. We present empirical results regarding the sensitivity of common metric definitions to various aspects of protocols such as recursion levels, multiple concurrent connections, transition or event patterns.
\end{abstract}

\section{Keywords}

Protocol testing, test selection, test coverage 


\section{INTRODUCTION}

Formal work on test coverage metrics for protocols had been long overdue when our metric based test selection method $[7,3]$ was first introduced; it provides a new, analytical way of assessing the quality of a test suite in terms of its coverage of the specification. Contrary to fault targeting models [1], where detection of a predefined fault classes (also called fault model [1]) is the test purpose, this metric based method seeks to achieve trace equivalence of the test suite with the specification, and therefore measures the coverage by examining how "closely" the test suite covers the specification.

The metric definition and the test selection method are interesting in that the former can be shown to lead to a compact metric space and the latter is tantamount to a convergent test selection process where the more test sequences are selected the closer the selected set tends to the orginal set, i.e. there are no relevant, peculiar test cases or groups of test cases that may be missed out in the selection process due to mere overlook, as are usually the cases in heuristic test selection. Furthermore, the metric defined is made general and flexible by a number of parameters which can be tuned according to the expert knowledge of the specific protocols and potential faults.

The definition of the metric, in terms of test distances, does not have to be related to fault detection capabilities of the test suite, since as long as the specification can be eventually covered, all faults that can occur should be discoverable during testing. However, the definition will certainly affect the effectiveness of the convergence process, since a "bad" definition of a test distance may make the process so slow that a large amount of test cases are needed to attain a desired coverage. The question so induced would be: how can one be sure that a metric is effective?

We looked at this problem, and believe that an effective metric should be able to capture important factors in a protocol specification, such as recursion levels, multiple concurrent connections, parallelism, and transition (or event) patterns, since they constitute major characteristics of a protocol. In other words, if a metric can incorporate these properties effectively, we can expect that it will effectively cover the specification with a reasonable amount of test cases. The difficulty, however, lies in analytically determining the effectiveness of a metric in handling them, since those properties may present themselves radically different in various protocols, and different metrics may be needed in different situations. We therefore resort to experimental methods that show the sensivity of common metrics to the properties.

In order to produce the results closer to real situations, we decide to use, in our experiment, a reasonably complex, real-life protocol, the Internet Stream Protocol [4] ${ }^{*}$, and a number of common metrics (to be defined later). Although the results may not be extrapolated to all other protocols and/or

*A recent revision of this protocol (ST2+, RFC 1819) is available within the internet community. 
metrics, we believe it can give us important initial results that help to design further experiments in assessing more metrics and protocols. With more results and additional "representative" real-life protocols, it will definitely help understand protocol properties and how a metric can capture them effectively.

The rest of the paper is organized as follows. We first give a brief overview of the metric based method, followed by a description of our experiment settings in Section 3. Section 4 presents our serial experiments on the sensivity of a metric to various properties. We conclude by discussing the observations and further research work.

\section{OVERVIEW OF THE METRIC BASED METHOD}

As already stated, the purpose of the metric based method is to generate test cases that cover the specification. A specification can be considered as describing the behaviour space of a protocol, where execution sequences constitute the control part. The whole space can be infinite, either an execution sequence can be infinite, or there are infinite number of execution sequences. Therefore, in order to cover the space within the computer system and time resources limit, approximations have to be made.

The metric based method solves this problem by defining a metric space over the behaviour space made of execution sequences. A set of finite execution sequences (a test suite) as approximations of infinite sequences, can be selected based on the metric. Furthermore, a finite number of test suites, which approximates the infinite behaviour space, can be generated based on the restriction of test cost. The important property of this approximation process is that the series of test suites can converge to the original specification in the limit. Thus, we have a way to achieve coverage of the specification with an arbitrary degree of precision limited only by the test cost.

The metric is built on the concept of test distance between two execution sequences. The distance satisfies the requirement that the resulting space be a metric space, so that we have the nice property of finite covers of an infinite space [3]. It should also grasp the intuitive requirement of testing relationships between execution sequences, so that a concept of "closeness" of sequences can be understood. This closeness actually represents a notion of testing representativeness: the closer the two sequences, the more likely they'll yield the same test result.

Formally, we define a test distance as [3]:

Definition. Let $s, t$ be two (finite or infinite) execution sequences in $S$ and let $s=\left\{\left(a_{k}, \alpha_{k}\right)\right\}_{k=1}^{K}$, and $t=\left\{\left(b_{k}, \beta_{k}\right)\right\}_{k=1}^{L}, K, L \in \mathbf{N} \cup\{\infty\}$. The testing distance between two execution sequences $s$ and $t$ is defined as

$$
d t(s, t)=\sum_{k=1}^{\max \{K, L\}} p_{k} \delta_{k}(s, t)
$$


where

$$
\delta_{k}(s, t)= \begin{cases}\left|r_{\alpha_{k}}-r_{\beta_{k}}\right| & \text { if } a_{k}=b_{k} \\ 1 & \text { if } a_{k} \neq b_{k}\end{cases}
$$

If $s$ and $t$ are of different lengths then the shorter sequence is padded to match the length of the longer sequence, so that $\delta_{k}=1$ for all $k$ in the padded tail.

Note that in the above definition, $p$ and $r$ are functions satisfying the following properties:

P1 $\left\{p_{k}\right\}_{k=1}^{\infty}$ is a sequence of positive numbers such that $\sum_{k=1}^{\infty} p_{k}=p<\infty$. P2 $\left\{r_{k}\right\}_{k=0}^{\infty}$ is an increasing sequence in $[0,1]$ such that $\lim _{k \rightarrow \infty} r_{k}=1$. Put $r_{\infty}=1$.

This definition guarantees that the space $(S, d t)$ is a metric space, and more importantly it is totally bounded and complete [3]. It ensures the existence of finite covers for infinite metric space $(S, d t)$, which is the theoretical foundation for the approximation process and also the test selection algorithm described below.

The selection algorithm for the metric based method (MB selection method for short), is a multi-pass process in which each pass is realized by the selection function SELECT $\left(T_{0}, G, \epsilon, C\right)$, which returns a selected set $T$, being an $\epsilon$-dense subset of the original set $G$ of test cases generated by some test generator, such that the cost of $T$ (which includes the initially selected set $T_{0}$ ) is less than some given threshold cost $C$.

The cost function of a test case can be defined to represent the resources (time and space) required to execute that test case, e.g. its length. The cost of a set of test cases can be defined simply as the sum of the cost of the individual test cases in the set.

\section{Metric-based test selection algorithm}

Step 1. Initially, the selected set $T$ is empty, $G$ is the given (generated) set of test cases, $\epsilon$ is the initial target distance, and $C$ is the given cost threshold for the selected set.

Step 2. While $\operatorname{Cost}(T)<C$ do $T=\operatorname{SELECT}(T, G, \epsilon=\epsilon / k, C)$ for some scaling factor $k>1$, applied at each iteration (that is, each pass).

Step 3. Stop. No further pass is possible because any other test case added to the set $T$ of test cases selected so far violates the cost constraint.

The function SELECT $(T, G, \epsilon, C)$ (that is, each pass in Step 2 of the above general algorithm) can be specified as follows:

Step $2.1 \quad$ Let $X=G \backslash T$, i.e. $G$ excluding $T$.

Step 2.2 If $X$ is empty return $T$ and exit, else remove (randomly) some test case $t$ from $X$. 
Step $2.3 \quad$ If $d t(t, T)<\epsilon$ goto Step 2.2 .

Step 2.4 If $\operatorname{Cost}(T \cup\{t\})<C$ then add $t$ to the selected set $T$.

Step 2.5 Goto Step 2.2.

As can be seen, the target distance $\epsilon$ decreases with each pass in the algorithm. The multipass algorithm generates Cauchy sequences, each being formed by test cases selected over successive passes. The Cauchy sequences converge to limits that are themselves in the original set of test cases, and any infinite test case in the original set can be approximated by some converging sequence of selected test cases. Thus, the algorithm performs test selection as a convergent approximation process, which ensures that no test cases or regions of test cases are overlooked.

\section{EXPERIMENT SETTINGS}

Having described the methodology of metric based test selection, we now look at the experiments we are going to do. The above algorithm selects test cases repeatedly until a certain test cost is reached. We can thus observe how test cases are selected with a certain test distance definition.

To get meaningful results, typical protocols and metric definitions should be used. However, there is generally no consensus on which protocols would be "typical". We therefore decide to use the Internet Stream Protocol, a protocol that is both practical (i.e., used in real applications) and interesting (i.e., relatively complex). We consider it as "typical" in the sense that it possesses interesting protocol properties as appeared in most real-life protocols. The results should therefore at least shed some light on similar protocols.

The Internet Stream Protocol has been used for years as the primary protocol in a voice and video conferencing system operating over the wideband and terrestrial wideband networks. This version of the specification was issued in 1990 , and is known as ST-II [4] *. The ST Control Message Protocol (SCMP) is mainly used in our experiments.

The basic functionality of SCMP is to build routing trees between a sender, called origin, and one or more receiving applications, called targets. One such routing tree will typically include one or more additional nodes along each of its paths, called intermediate agents. ST (the data phase) then simply transmits data down the routing tree, from an origin to one or more targets. The data flow over the routing tree is referred to as a stream. Each of the communicating entities within a routing tree runs an ST-II protocol and is

"Resource Reservation Protocols (of which ST-II is one representative) are an important class of protocols that is currently receiving undivided attention of the internet community, and other communications forums concerned with real-time streaming (multimedia) protocols. 
called an ST-II agent. One ST-II agent can simultaneously act as one or more origins, routers, and targets.

From the testing point of view, the ST-II protocol makes it interesting for the following reasons:

1. There are two different sources for the concurrency in ST-II: a) multiple protocol connections, and b) simultaneous execution of origin, target, and intermediate agent functional entities within one ST-II agent.

2. With up to 17 different types of messages for SCMP part of ST-II, STII becomes one of the more complex protocols, especially if the combined state space composed of one origin, one target, and one intermediate agent module is considered.

3. The protocol is non-symmetrial since individual connections are trees. The usual concept of a "peer" partner in communication, as a convenient symmetrical counterpart in testing, has to be abandoned.

We carried out the experimentation under the assumption that an STII protocol implementation is tested as an entity, composed of independent parallel executions of (several) origin, intermediate or target agents. Because of the non-symmetricity of the communication pattern, the temporal order of protocol primitives arriving from IUT as a response to different upstream or downstream agents (even in one-stream environments) is unpredictable and should be abstracted. Therefore, the test architecture applicable in this setting is the interoperability test architecture, where a set of lower PCO-s must be observable.

\subsection{Test development system}

The test system that we use to conduct the experiments is the TESTGEN+ system [8]. A functional diagram of the system is shown in Figure 1.

\section{The TESTGEN Module}

TESTGEN [8] is the test generator module in an experimental protocol TEST Generation ENvironment for conformance testing developed at the University of British Columbia. The generated test suites incorporate both control flow testing and data flow testing with parameter variation. Both types of testing are controlled by a set of user-defined constraints which allows a user to focus on the protocol as a whole or just on restricted areas of the protocol.

TESTGEN begins with a protocol description in an extended transition system formalism (a subset of Estelle [5]) and ASN.1 description of the input service primitives, output service primitives, and protocol data units. Once all constraints are defined, TESTGEN identifies subtours within the specified 


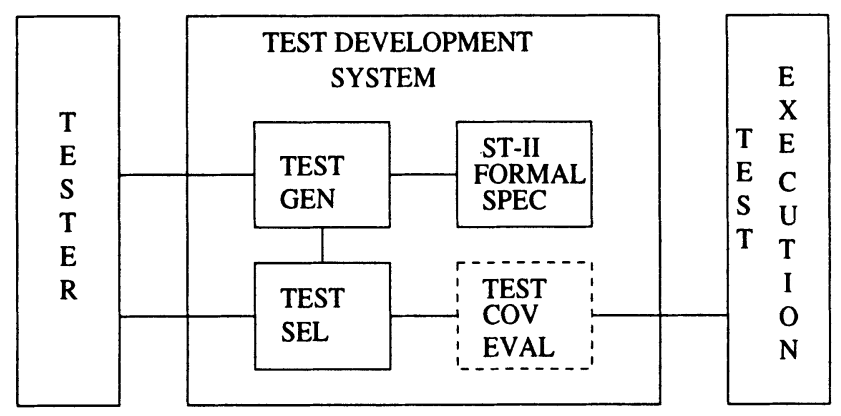

Figure 1 The Functional Structure of the Test Development System.

protocol which satisfy the minimum and maximum usage constraints. Each subtour then undergoes the parameter variation defined by the types of service primitives in the subtour and the parameter variation constraints.

\section{The TESTSEL Module}

The TESTSEL module (which optionally includes an independent test coverage evaluator TESTCOV EVAL module), is an implementation of test case selection (as presented in in Section 2) and test case coverage evaluation based on the distance and coverage metrics within the metric-based method. Since the test coverage metric we are using is guaranteed to be convergent when Cauchy sequences are followed, and the algorithm produces such sequences, a set of test cases which converges to the initial test suite will be iteratively yielded.

The two modules, TESTGEN and TESTSEL have been integrated into a test development environment similar to the one already used for the development of the InRes protocol test sequences [6]. The seed files for test suites used in experimentation are generated by TESTGEN. These can be fed into the TESTSEL module directly, or first passed through an independent interleaving algorithm. This algorithm produces random independent interleavings and random recursive levels of a specified number of streams and target agents, in order to obtain test suites exercising multiple simultaneous connections, concurrency of different agents, and higher levels of recursion. The output of the TESTSEL module and the random interleaving algorithm are then compared and analysed.

\section{EXPERIMENT RESULTS}

We first developed an Estelle specification of the ST-II origin, intermediate, and target agents (refer to Appendix E of [2] for details), and an ASN.1 description of the data part. The specifications are then fed to our TESTGEN system to generate the sets of subtours. These sets are named originseeds, 
intermediateseeds, and targetseeds, corresponding to the origin, intermediate, and target agents. The purpose of these sets is to serve as the starting sets for the random interleaving and recursion generation process that we implemented specifically for the use in the experiments. They should preferably provide a thorough coverage of the relevant tours through each individual agent's state space in their simplest form (i.e., without concurrency or recursion).

The random independent interleaving module generates test suites with concurrency of: a) multiple streams (simultaneous connections) within the same ST-II agent; b) multiple agent functional entities within the same ST-II agent; and c) multiple targets within the same stream. Concurrency due to the interleaving of multiple intermediate agents within the same stream has been omitted. In the experiments, we examine the effects of the recursion and concurrency of the ST Control Message Protocol only, since the data phase of this protocol is trivial.

\subsection{Stability and granularity of the test selection process}

The next two experiments investigate the granularity and stability of the test selection process guided by a typical metric function.

The metric function $p_{k}$ takes values $p_{k}=2^{10-k}$, for $k=1,2, \ldots$. The function $r_{k}$ takes values $r_{k}=1-\frac{1}{2^{k}}$ for all $k=1,2, \ldots 20$ sets of 100 test cases were generated, representing random interleavings of 1 through 10 simultaneous network connections, and 10 through 80 ST-II target agents. Test sets are labelled tsc.t, indicating the number of simultaneous connections $c$ and targets $t$ that were allowed in their generation.

\section{Experiment 1.1}

The first series of 10 test sets contained test cases with individual events labelled by event names only. (This would be suitable for testing event variations at global levels, e.g. does an implementation correctly react to an event $e$ after some event $d$.) Figure 2 shows the number of test cases selected versus refinements in the test density. $x$ axis represents the density range of 0 through 1024.00, the diameter of the metric space generated by this metric. For this metric, results for ts 10.35 and ts 10.50 coincide. Also, the line styles of ts 10.30 and ts10.35 are reused for ts 1.80 and ts2.70 - the lower lines belong to ts1.80 and ts2.70.

\section{Experiment 1.2}

The second series of 10 test sets contained test cases with individual events labelled by event names, transition tour they belong to and the stream identifier. (This is a much finer identification which would allow detailed testing of event variations within individual connections and with respect to event 
parameter data variations). Figure 3 shows the granularity of the selection process for this case.

In both cases, i.e. given both very fine (many distinct event labels), and much coarser (relatively few distinct event labels) event space, there is a steady increase in the number of test cases selected as the density approaches 0 . Figures at the low end of density spectrum indicate, that even at very fine granularity levels of density, this metric function is still capable of distinguishing still finer test suites. More test cases are selected for test suites in the second series of tests, for the same levels of density, number of simultaneous connections and recursion, since more distinct event labels can be identified. In both graphs, ts2.60 and ts2.70 occupied the middle portion of the graph, with the suites ts1.t (ts5.t and ts10.t) almost entirely below (above, resp). Given same density levels and event labelling, fewer test cases are almost always selected for test suites involving fewer simultaneous connections, indicating a poorer event pattern. At the same time, given the same number of simultaneous connections, the effect of higher vertical recursion (more targets) on the number of test cases selected is not very noticeable, given the low values of the $r_{k}$ series for moderate values of $k$ in this example.

The stability and granularity of the MB selection which are observed in these two experiments, are confirmed throughout the test selection and coverage analysis experiments that follow.

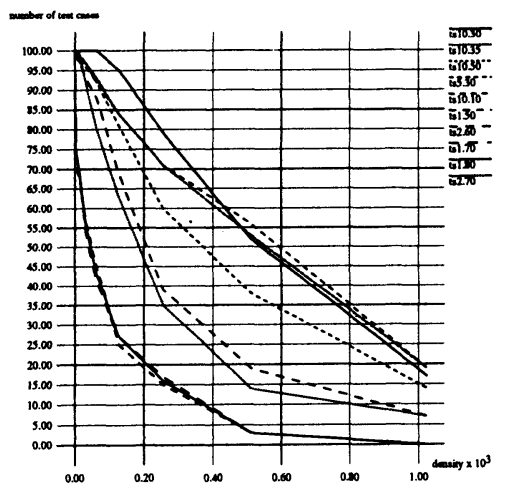

Figure 2 Granularity of MB test selection with event name identification.

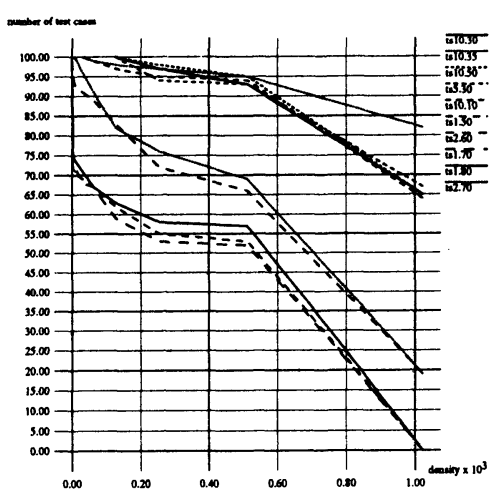

Figure 3 Granularity of MB test selection with event name, gate and data parameters identification.

\subsection{Identifying transition tours}

\section{Experiment 2}


This experiment investigates the efficiency of applying metric based method for transition tour selection, for single connection environments, with multiple agent functionality and with moderate vertical recursion. We allow three different Finite State Machines to represent individual connections.

We use the seed files, i.e., originseeds, intermediate seeds, and targetseeds, for each of the three agents of the ST-II protocol machine. We hypothesize that they identify all transitions of each agent. We did not have a tool to generate or verify the transition tours, consequently the value of the test is in the efficiency estimate rather than the exactness of the transition tour identification.

The metric used is $p_{k}=2^{9-k}$. Also, the recursion was given a minimal weight through the $r$ function as in experiments 1.1 and 1.2.

Let $N_{T}$ be the minimum level of the reachability tree corresponding to the specification by which all transitions have been traversed by a subtour. We observe that the largest $N_{T}$ is for the intermediate agent, where $N_{T}=9$, for ACK packets to DISCONNECT or REFUSE PDU-s. We generated 8 test suites, containing randomly selected one-connection environments with the upper bound on vertical recursion (number of targets) equal to 30. All transition tours from seed files were added to these sets in order to simulate test suites which have transition coverage. Generated test suites had 177, 277, $377,577,677,777,877$ and 977 test cases, and are designated ts.n, where $n$ is the number of test cases they contained. Figure 4 (enlarged portion in Figure 5) shows the number of test cases selected by the progressive passes of the test selection algorithm, for each of the test sets, as the density covers the range from 511 to 0.998047 . At 0.998047 the algorithm is guaranteed to have identified transition cover of the three agents, when they work in isolation, provided the experiment's hypothesis is satisfied.

The greater variety in event patterns and recursion is expected, as the randomly generated test sets grow larger. This accounts for more test cases selected, at equal density levels, for larger test sets.

The results show that, even with larger test suites, the algorithm is not overly wasteful, when used for identifying test suites with fault coverage at least equal to that of the transition tour method. Since the metric used in the experiment yields a complete metric space, the experiment simulates a test selection environment where, first, a test suite of transition tours equivalent coverage is selected, after which the process further proceeds by identifying still finer subsets of the initial set, with respect to variations in patterns of recursion and event sequencing. Completeness guarantees complete coverage of the initial set in the limit.

\subsection{Sensitivity to vertical recursion}




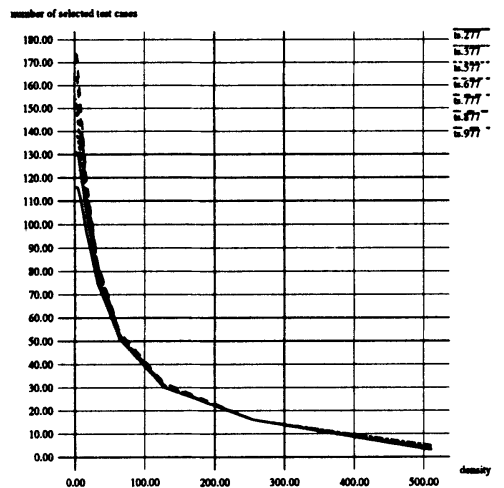

Figure 4 Selecting Transition Tours with moderate vertical recursion.

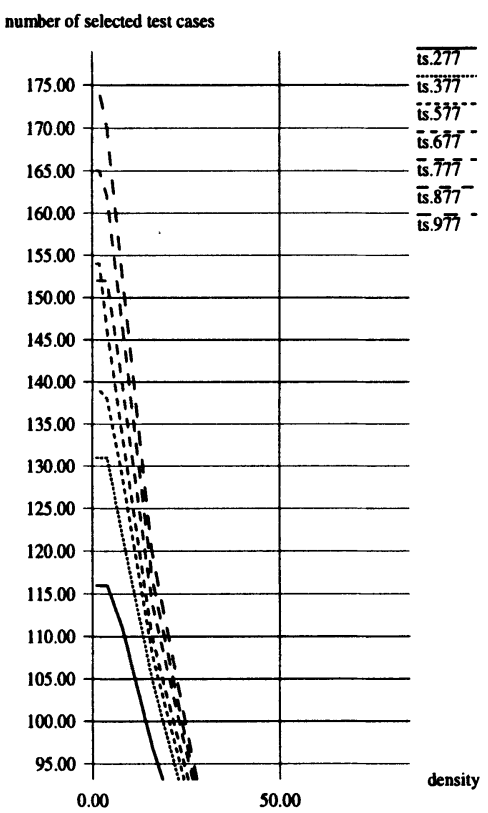

Figure 5 Selecting Transition Tours with moderate vertical recursion.

In this experiment we investigate the effect of vertical recursion on the mutual density of test sets. The metric function used is the same as in the previous experiment. We use test suites of 100 test cases each, randomly generated, with low numbers of simultaneous connections $(1-3)$, and moderate vertical recursion $(10-30)$ targets. The experiment shows that sets generated with a certain number of simultaneous connections and a certain event pattern are less dense in the sets with the same characteristics, if they contain less recursion than those sets.

Figure 6 shows this effect of vertical recursion on mutual coverage of two series of 6 test suites each. Sets $r_{1}, \ldots, r_{6}$ are randomly generated sets of test cases, and $s_{1}, \ldots, s_{6}$ are their corresponding sets (same number of simultaneous connections and event patterns), but with a certain reduction in recursion when calculated with respect to the corresponding set (i.e. the same subscript) from the $r$ series. The test suite generation specifics and the amount of reduction in recursion (in percentages) are shown in Table 1. No special effort was taken to fairly space the recursion levels in either $r$ or $s$ series of test sets.

The effect is plotted by representing the mutual density for each pair of sets $\left(r_{k}, s_{k}\right), k=1, \ldots, 6$ running along the $x$-axis. Connecting the points of the scatter graph allows for easier viewing. The density points of sets $s_{1}, \ldots, s_{6}$ 
Table 1 Experiment 3 - Characteristics of test sets.

\begin{tabular}{lccr}
\hline set & connections & targets & rec. reduction for sets $s_{k}$ \\
\hline r1 & 1 & 10 & 30 \\
r2 & 2 & 10 & 50 \\
r3 & 3 & 30 & 100 \\
r4 & 1 & 30 & 25 \\
r5 & 2 & 30 & 100 \\
r6 & 3 & 20 & 70 \\
\hline
\end{tabular}

in sets $r_{1}, \ldots, r_{6}$ are connected by the $r / s$ line, and the density points of sets $r_{1}, \ldots, r_{6}$ in sets $s_{1}, \ldots, s_{6}$ are connected by the $s / r$ line.

We generally found that with random generation, sets of 100 test cases were sufficient, under given protocol characteristics, for the mutual coverage figures to show sensitivity towards vertical recursion at reduction levels in the range of 25-100 percent. This is due to the fact that, given a sufficient number of test cases in a test suite and a test generation algorithm that does not necessarily cluster recursive events in few clusters with poor distribution, lower bounds of recursion are more likely to generate less variety in event cluster recursion. Consequently, it will be easier for the set which has higher bounds on recursion to approximate event patterns and recursive levels of poorly recursive sets than vice versa, under such conditions.

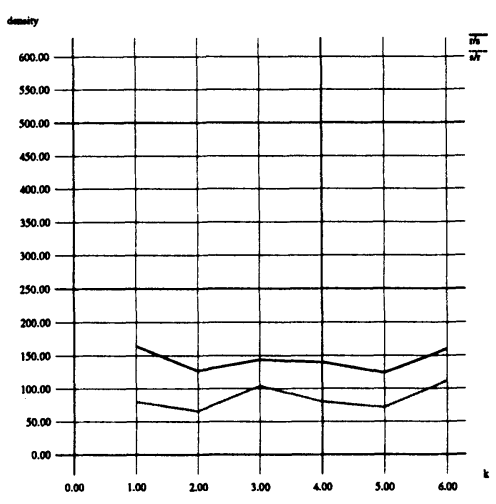

Figure 6 Sensitivity to vertical recursion.

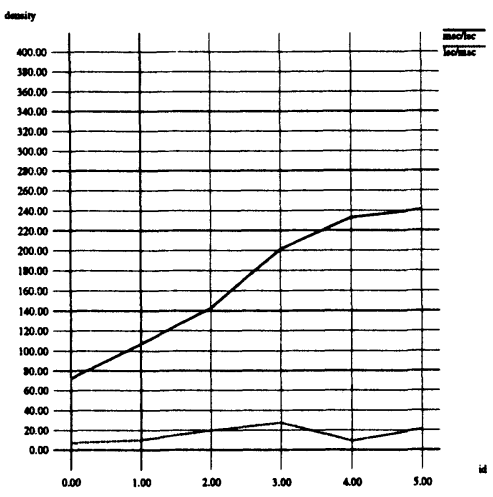

Figure 7 Mutual densities of some sets with moderate and low number of simultaneous connections.

In a related experiment (not shown here), pairs of randomly generated sets, with moderate numbers of simultaneous connections, one with low and one 
with high recursion, were compared for mutual coverage. Under the same metric function as in this experiment, the results were inconclusive. We identified the cause by examining sequences which contributed to high maximum distances. We concluded that a new event pattern in a test sequence, likely to appear early in a sequence with many simultaneous connections, could easily offset the effect of better recursion distribution under this metric.

Joint conclusion from both experiments is that the quality of test suite expressed through metric based coverage functions is sensitive towards vertical recursion, however a metric with adequate sensitivity to recursion should be used if it is to be a decisive factor of the quality of test suite.

\subsection{Sensitivity to parallelism}

\section{Experiment 4}

In this experiment we investigate the impact of testing with low and moderate network connection parallelism on the mutual coverage of test suites that differ with respect to the level of parallelism. The experiments were conducted under quite general circumstances. All test sets, 6 with low and 6 with moderate parallelism of network connections, were randomly generated, containing 100 test cases each. Therefore a certain potential existed for inconclusive results due to choosing a particularly rare combination of events in either case. However, the mutual coverage results were consistent in 3 different metric spaces and throughout 36 different comparisons. We therefore concluded that the results were a good indication of the MB coverage function to identify suites with greater simultaneity of network connections, in a general case. The results are reported in Figures 7, 8, 9.

The characteristics of individual test suites, with respect to the number of simultaneous connections and the number of targets are given in Table 2. Suites involved moderate vertical recursion (10-30 targets), and were put into "low simultaneous connection" (lscid) set if involving 1 to 3 simultaneous connections. Likewise, "moderate simultaneous connection" sets (mscid) involved 10 - 35 simultaneous connections. Suites with equal $i d$-s were compared.

The comparisons were carried out in metric spaces generated by metrics with

1. $p_{k}=1$, for $k<400$, and $p_{k}=\frac{1}{2^{k-400}}$, for $k \geq 400$.

2. $p_{k}=1$, for $k<25$, and $p_{k}=\frac{1}{2^{k-25}}$, for $k \geq 25$.

3. $p_{k}=1$, for $k<15$, and $p_{k}=\frac{1}{2^{k-15}}$, for $k \geq 15$.

and the series $r_{k}$ were defined as in Experiment 1.2.

The plots for each of these metric spaces are in Figures 7, 8, 9, respectively. $X$-axis is labelled with set $i d$-s, from 0 through 6 . The plots show the density of sets $l s c 0, \ldots, l s c 5$, in sets $m s c 0, \ldots, m s c 5$, resp. These scatter points are 
Table 2 Experiment 6 - Characteristics of randomly generated test sets.

\begin{tabular}{lrrr}
\hline test set id & connections & targets & cases generated \\
\hline $\mathrm{msc} 0$ & 10 & 30 & 100 \\
$\mathrm{lsc} 0$ & 1 & 30 & 100 \\
$\mathrm{msc} 1$ & 15 & 30 & 100 \\
$\mathrm{lsc} 1$ & 1 & 30 & 100 \\
$\mathrm{msc} 2$ & 20 & 30 & 100 \\
$\mathrm{lsc} 2$ & 2 & 30 & 100 \\
$\mathrm{msc} 3$ & 25 & 10 & 100 \\
$\mathrm{lsc} 3$ & 3 & 30 & 100 \\
$\mathrm{msc} 4$ & 30 & 10 & 100 \\
$\mathrm{lsc} 4$ & 1 & 10 & 100 \\
$\mathrm{msc} 5$ & 35 & 10 & 100 \\
$\mathrm{lsc} 5$ & 2 & 10 & 100 \\
\hline
\end{tabular}

connected by a line labelled msc/lsc. Similarly, the line labelled lsc/msc connects points that show densities of sets $m s c 0, \ldots, m s c 5$ in sets $l s c 0, \ldots, l s c 5$, resp.

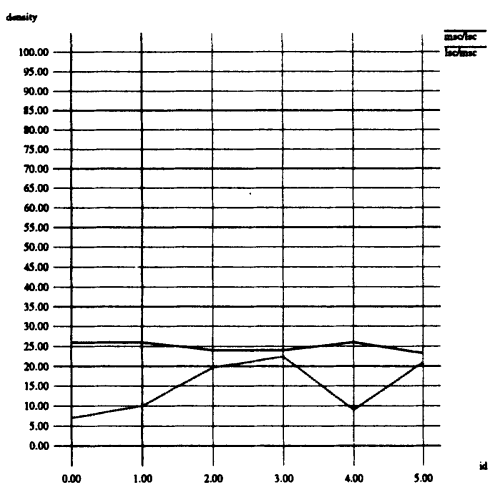

Figure 8 Mutual densities of some sets with moderate and low number of simultaneous connections.

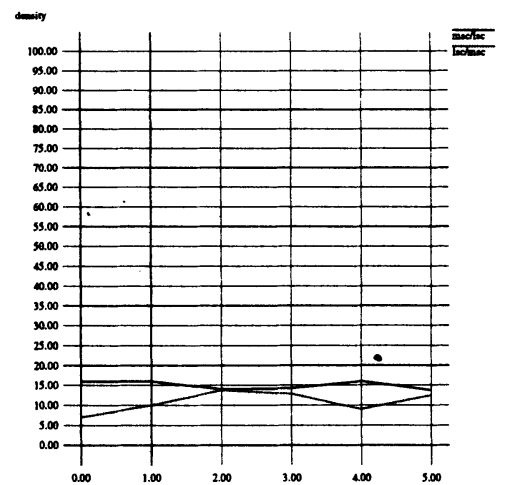

Figure 9 Mutual densities of some sets with moderate and low number of simultaneous connections.

Although the test sets with moderate parallelism are consistently more dense in low-parallelism sets in all three metric spaces considered, significant difference exists in the magnitude of the mutual density variation. Metric 1 evaluates all patterns of very long sequences with equal value, up to a large $k=400$. Therefore, the density variation in the space generated by this 
metric can be attributed to the much larger length of sequences involving many connections. This, however, is a definite indication of the number of connections exercised, given fixed vertical recursion, but not necessarily of the simultaneity of such connections. Therefore, the next two metric spaces favoured the average and upper end case length of sets lscid, which was found to be in the range of $11-36$. (Lengths of mscid test cases were in the range of 60 - 145.) Consequently, they really evaluated the greater variation in pattern of test sequences generated by many simultaneous connections, especially in view of the fact that all sequences longer than 15 (25) would contribute at most 1 to the final distance. This is an extreme case which definitely did not give a priori advantage to longer test sequences of sets mscid. It is therefore conceivable that a more moderate metric would yield results consistent with this experiment's results.

\subsection{Sensitivity to a missing event or event combination}

The purpose of the next two experiments is to investigate the sensitivity of mutual densities of two test suites, when one of the test suites does not contain an event or event combination, or does so only to a certain lesser extent. All test sets contain 100 test cases. Metric is the same as in Experiment 1.1, unless otherwise noted.

\section{Experiment 5.1}

First, the sensitivity analysis was carried out for the environments involving no parallelism and moderate vertical recursion. In this experiment, 20 randomly generated sets of 100 test cases each were compared for mutual coverage. Test sets $s_{1}$ through $s_{10}$ are random interleavings of one connection, up to 30 ST-II targets. Test sets $o_{1}$ through $o_{10}$ are random interleavings of one connection, up to 30 ST-II targets, where ST-II agent origin does not include sequences with a PDU HAO (HID APPROVE) as a response to a CN (CONNECT) PDU. Figure 10 shows the density deviation for the densities of sets $o_{k}$ in randomly generated test sets $s_{k}$, connected by the line s/o. For comparison, also shown are plots of all other density figures available in the experiments: a) densities of sets $s_{k}$ in sets $o_{k}$, connected by a line o/s, b) densities of sets $s_{k}$ in sets $s_{k+1}$, connected by a line s/s, and c) densities an oracle set originoracle in sets $o_{k}$, line o/originoracle.

\section{Experiment 5.2}

In this experiment we investigated environments with moderate parallelism and moderate vertical recursion. This experiment shows the effects of a missing event (SP or PDU) in a more complex test suite. 3 groups of test sets, 100 random test cases each, were generated to randomly contain between 5 to 10 simultaneous connections, and 10 to 30 targets. The set $s_{1}$ through $s_{6}$ 
was generated taking into account all available SPs and PDUs for all ST-II agents. Sets $r_{1}$ through $r_{6}$ were generated with DSI (DISCONNECT PDU from the direction of origin) missing from intermediate seed sets. Likewise, sets $t_{1}$ through $t_{6}$ were generated with HCT PDU missing from target agent seed sets.

Figure 11 shows that applying general metric to mutual coverage of such sets yields inconclusive results. Lines $s / r, r / s, s / t$ and $t / s$ connect scatter points of densities of sets $r, s, t$, and $s$ in sets $s, r, s$ and $t$ respectively, where densities are calculated only between sets with equal subscripts.

We did observe, however, that at these levels of parallelism and recursion, the mutual coverage of randomly generated test sets with all events included into seed files, consistently showed better density figures.

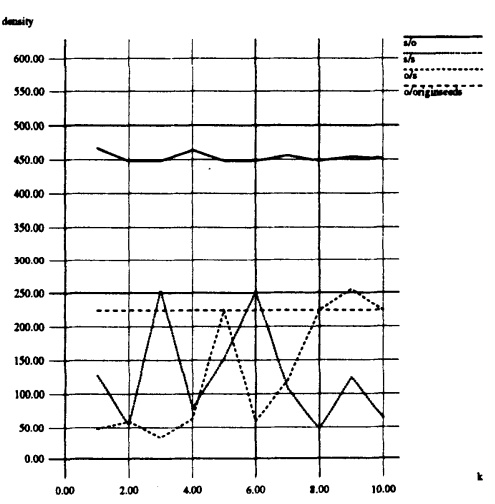

Figure 10 Sensitivity to missing events - single connection with recursion.

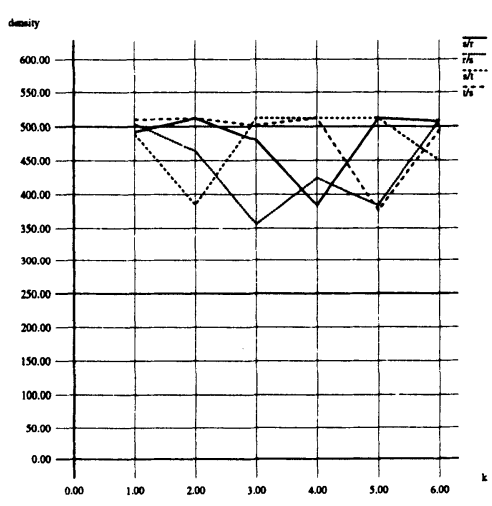

Figure 11 Sensitivity to missing events - general metric function, multiple connections with recursion.

In a related experiment, we improved sets $t$ from the previous experiment, by adding 10 percent and 70-80 percent of sequences, involving the HCT PDU. Figure 12 shows the range of densities of sets $t_{1}, \ldots, t_{6}$ (all HCT event sequences deleted), $t_{1_{\text {aehct }}}, \ldots, t_{6_{\text {aehct }}}$ with almost all HCT events missing (10 percent sequences with it), and $s_{1}, \ldots, s_{6}$ with $70-80$ percent sequences with HCTs (which is the typical content in a randomly generated test set of these characteristics), in randomly generated test sets $r s_{1}, \ldots, r s_{6}$, applying a general metric function (from Experiment 1.1).

Although the results explain the densities in Figure 11 to a certain extent, it still would be necessary to have a knowledge of the expected densities at particular levels of vertical recursion and parallelism, in order to use general metric functions for missing event identification.

In the following experiments, a special metric function was designed to identify the coverage of a particular event (HCT PDU in this case). Its definition is 
the same as in Experiment 1.1, except for the cases were neither of the events at a position $k$ in two sequences is HCT: if this is the case, the summand $p_{k} \delta_{k}$ for this position $k$ is zero. This metric function measures the coverage of the event HCT both with respect to its position in sequences and the level of recursion.

Figure 13 shows the application of the metric function to specially identify the coverage of HCT event, and its improvement with improving t-test sets with sequences containing event HCT, up to its average 70-80 percent representativeness as observed in randomly generated test sets. These are calculated as densities of $t$ sets in randomly generated s sets.

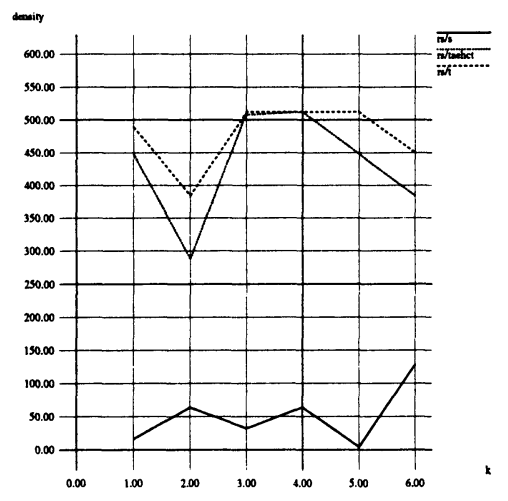

Figure 12 Sensitivity to missing events - general metric function, moderate parallelism and recursion.

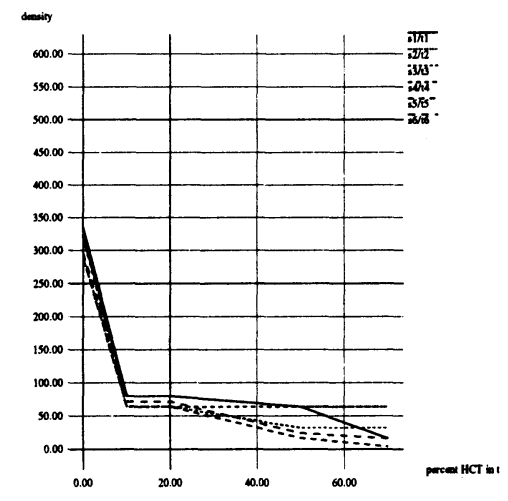

Figure 13 Sensitivity to missing events - special metric, moderate parallelism and recursion.

Figure 14 shows the effect of improving sets $t_{1}$ to $t_{6}$ with $10,20,50$ and 70 80 percent of sequences which contain HCT in case of general metric. These are calculated as densities of $t$ sets in randomly generated $s$ sets. These do not improve as fast as in the previous metric.

All subsequent examples use the special metric designed to identify missing event content. The following figures show the sensitivity of this metric in various situations.

Figure 15 shows the gain in the quality of $\mathrm{t}$ by increasing its HCT content, by plotting the density of randomly generated test suites, in sets tid, whose special event content (HCT content) is improved by adding $10-70$ percent test sequences involving the event considered.

The next 2 figures show how the plots for 2 combinations of randomly generated test sets $\mathrm{s}$ and sets $\mathrm{t}$ from this same example, meet in the range of 16-64, as the coverage of the event HCT increases. (These are excerpts for fixed combinations of test sets from Figures 6.17 and 6.20.)

Figure 18 shows mutual coverages of the randomly generated sets and set 


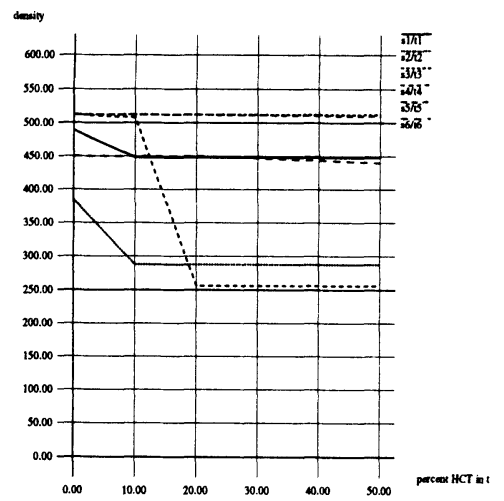

Figure 14 Sensitivity to missing events - general metric function, moderate parallelism and recursion.

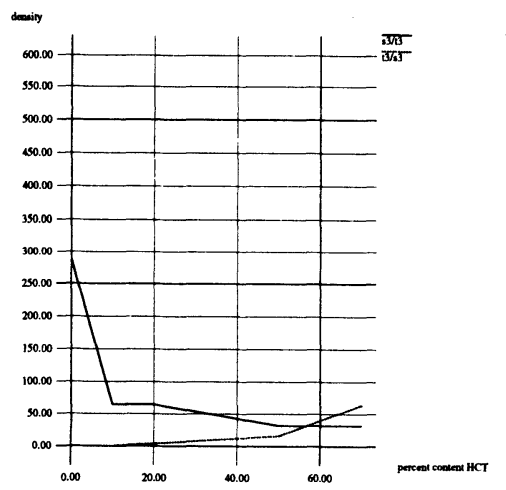

Figure 16 Sensitivity to missing events - special metric, moderate parallelism and recursion.

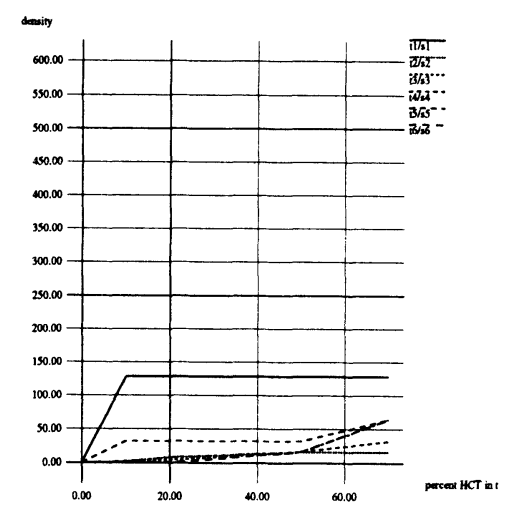

Figure 15 Sensitivity to missing events - special metric function, moderate parallelism and recursion.

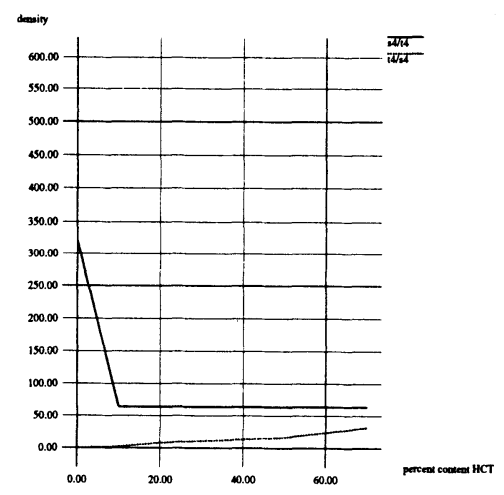

Figure 17 Sensitivity to missing events - special metric, moderate parallelism and recursion.

combinations in the same metric. Sets typically include $70-80$ percent tests with HCT. Random sets were generated using 5-10 connections, 10-30 targets. Experiments show that in such cases, mutual coverages of randomly generated sets also tend to settle in this same range.

\section{CONCLUSIONS}

We have performed a series of experiments to explore the metric based method's ability to identify or select test suites with certain levels of vertical recursion, 


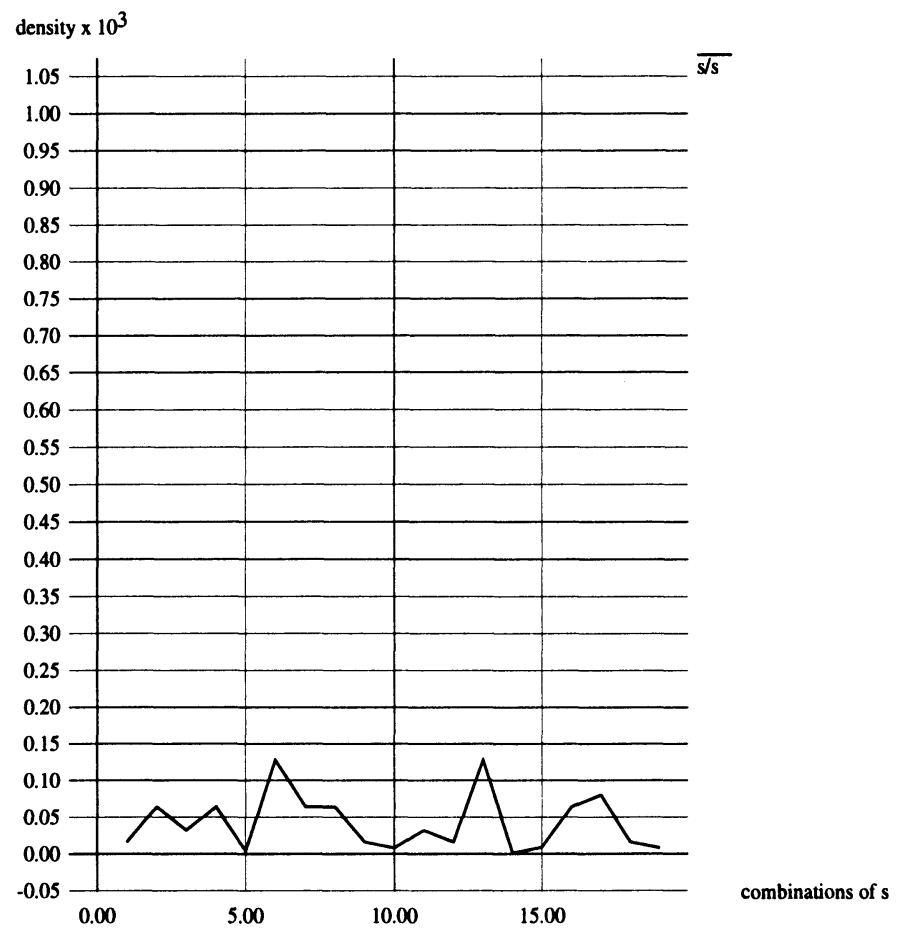

Figure 18 Sensitivity to missing events - special metric on random sets.

multiple connection capabilities, and transition or event patterns. The results show that the metric functions were stable and able to handle fine granularity of protocol behavior. The metric functions are shown to be sensitive to both recursion and parallelism, which are often primary sources of complexity in protocol specifications. The effect of missing events or event patterns with different metric functions and moderate parallelism and recursion were also observed.

In this empirical study, we have endeavored to provide a reasonably complete assessment of the sensitivity of the method by applying it to a fairly complex, real-life protocol, the ST-II protocol, which belongs to a class of protocols currently in the center of interest for multimedia streaming communications and the networking communities in general. It also exhibits most sources of protocol complexities, including recursions and multiple concurrent connections. We find the test selection method to be empiritically attractive in general. However, for a more thorough investigation, further experiments can be conducted for other "representative" complex protocols, with other metrics and even larger sets of test suites. Obviously, a fair amount of work would 
be demanded for each set of sensitivity assessment experiments performed on each additional protocol.

Further work may also include more experiments from which heuristics could be extracted to guide the choice of metric functions. Last, but not least, we may also study the sensitivity of metric functions to fault detection capability of the test suite generated. This is intuitively intriguing since by covering the specification sufficiently, faults should have a good chance to get detected.

\section{REFERENCES}

[1] G.v. Bochmann and et al. Fault models in testing. In J. Kroon, R.J. Heijink, and E. Brinksma, editors, Protocol Test Systems, V, Leidschendam, The Netherlands, October 1991.

[2] J.A. Curgus. A metric based theory of test selection and coverage for communication protocols. PhD thesis, Dept. of Computer Science, Univ. of British Columbia, June 1993.

[3] J.A. Curgus and S.T. Vuong. A metric based theory of test selection and coverage. In Proc. IFIP 13th Symp. Protocol Specification, Testing, and Verification, May 1993.

[4] C. Topolcic (ed). Experimental internet stream protocol, version 2 (STII); RFC-1190. Internet Requests for Comments, (1190), October 1990.

[5] Y. Lu. On TESTGEN, An Environment for Protocol Test Sequence Generation, and Its Application to the FDDI MAC Protocol. Master's thesis, The University of British Columbia, 1991.

[6] M. McAllister, S. T. Vuong, and J. Curgus. Automated test case selection based on test coverage metrics. In Protocol Test Systems V. Elsevier Science Publishers B.V. (North Holland), 1992.

[7] S.T. Vuong and J.A. Curgus. On test coverage metrics for communication protocols. In Proc. 4th Int. Workshop on Protocol Testing System, 1991.

[8] S.T. Vuong, H. Janssen, Y.Lu, C. Mathieson, and B. Do. TESTGEN: An environment for protocol test suite generation and selection. Computer Communications, 17(4):257-270, April 1994. 